\title{
Interesse e conhecimento em cirurgia refrativa entre estudantes de medicina
}

\author{
Interest and awareness in refractive surgery among medical students
}

\author{
Flávio Cotait Kara José ${ }^{1}$ \\ Lúcia Battistella Passos ${ }^{2}$ \\ Ana Carolina Jervásio ${ }^{2}$ \\ Gustavo Henrique Salomão ${ }^{3}$
}

\footnotetext{
${ }^{1}$ Acadêmico do segundo ano da Faculdade de Medicina do ABC - São Paulo.

${ }^{2}$ Acadêmico do terceiro ano da Faculdade de Medicina do ABC - São Paulo.

Acadêmico do sexto ano da Faculdade de Medicina do ABC - São Paulo.

Orientador: Prof. Dr. Newton Kara José. Professor titular da disciplina de oftalmologia da Faculdade de Medicina da USP e da Faculdade de Medicina da UNICAMP.

Endereço para correspondência: Rua Vicente Dorsa, 85 São Paulo (SP) CEP 04648-090.

E-mail: luciabpassos@hotmail.com

Recebido para publicação em 30.11.2000

Aceito para publicação em 20.08.2001
}

\section{RESUMO}

Objetivo: Realizou-se um estudo em estudantes da Faculdade de Medicina do $\mathrm{ABC}$ a fim de analisar e relacionar a freqüência de usuários de correção óptica, o tipo de vícios de refração, o número de pessoas submetidas à cirurgia refrativa e o conhecimento e interesse por essa operação. Métodos: realizou-se um levantamento entre estudantes de medicina da Faculdade de Medicina do ABC, no período de 19 a 21 de junho de 2000. Foi utilizado um questionário auto-aplicável como instrumento de coleta de dados. Resultados: foi relatado que $62,7 \%$ dos estudantes usavam correção óptica, sendo que 53,3\% desses apresentavam, como erro de refração, miopia simples ou associada ao astigmatismo. $92,8 \%$ do total dos entrevistados já tinham ouvido falar em cirurgia refrativa, contendo apenas $34,2 \%$ conhecedores dessa técnica cirúrgica e $17,6 \%$ conhecedores dos riscos e complicações pós-operatórios. Entre os 200 estudantes amétropes, 50,5\% gostariam de ser submetidos à operação, sendo que 69,0\% deles esperavam, através da cirurgia, a cura definitiva. Foi coletado, também, que 51,7\% dos entrevistados tiveram a última consulta oftalmológica há menos de 1 ano; $32,0 \%$ entre 1 e 3 anos e 15,7\% há mais de três anos. Apenas 5 estudantes já tinham sido submetidos à cirurgia refrativa. Conclusão: A maioria dos estudantes de Medicina (62,7\%) é portador de vício de refração corrigido, sendo os mais freqüentes a miopia simples e a miopia associada a astigmatismo. Há pouco conhecimento e falsa expectativa em relação à cirurgia, sendo que apenas $34,2 \%$ entrevistados conhecem o procedimento cirúrgico, $17,6 \%$ sabem dos riscos e das complicações e $69,0 \%$ esperam cura total. Diante das condições desse estudo, foi constatado que apesar de muitos se interessarem pela cirurgia refrativa, poucos se submeteram a ela, devido, principalmente, em ordem decrescente, a: contra-indicação médica, falta de oportunidade, falta de conhecimento e problemas financeiros. Os consultórios oftalmológicos são apenas a quarta fonte de informação, precedido, em ordem decrescente, por familiares, amigos e faculdade, o que é evidenciado pelo fato de $81,5 \%$ dos entrevistados gostariam de se informar melhor sobre a cirurgia refrativa. Mesmo tendo $83,7 \%$ se consultado nos últimos três anos, informações e esclarecimento satisfatório não foram fornecidos.

Descritores: Refração ocular; Erros de refração/cirurgia; Conhecimentos, atitudes e prática; Estudantes de medicina

\section{INTRODUÇÃO}

Os vícios de refração constituem a principal causa da diminuição da acuidade visual e atingem, de maneira crescente, todas as faixas etárias. A correção óptica, aos 60 anos de idade, é necessária em cerca de 100\% dos $\operatorname{casos}^{(1)}$. Óculos são a principal opção de correção óptica e, muitas vezes, a 
única possível. Por outro lado, a partir da década de 1960, o uso de lente de contato vem aumentando no mundo todo, chegando a aproximadamente $22 \%$ das correções ópticas nos

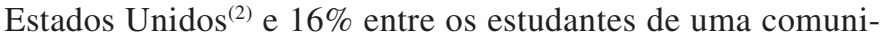
dade universitária no Brasil ${ }^{(3)}$.

Há muitas décadas procura-se uma correção definitiva nos casos de ametropia. A introdução da ceratotomia radial ${ }^{(4)}$, em 1933, trouxe grande expectativa. Novas pesquisas culminaram com o desenvolvimento da cirurgia e o uso de excimer laser em $1983^{(5)}$, nos EUA ${ }^{(2)}$. O número de cirurgias com excimer laser tem aumentado graças ao desenvolvimento de equipamentos que possibilitam resultados mais precisos.

A população entre 20 e 40 anos de idade é a que mais se beneficia com essa cirurgia, pela possibilidade de ficar livre do uso de óculos até o momento da presbiopia que tem sua maior incidência a partir dos 40 anos de idade ${ }^{(6)}$. Em um dos poucos trabalhos sobre prevalência de uso de correção óptica no Brasil foi constatado o uso de óculos ou lente de contato em 56,7\% dos estudantes da Universidade Estadual de Campinas $^{(3)}$ e nenhum caso de cirurgia refrativa entre eles.

O presente trabalho avalia, em estudantes da Faculdade de Medicina do $\mathrm{ABC}$, a freqüência de usuários de correção óptica, o tipo de vício de refração, o número de submetidos à cirurgia refrativa, além do conhecimento e do interesse por essa cirurgia.

\section{MÉTODO}

Foram entrevistados 319 estudantes do curso de Medicina do $1^{\circ}$ ao $4^{\circ}$ ano da Faculdade de Medicina do ABC (Santo André, SP), no período de 19 a 21 de junho de 2000 que estavam presentes no local no momento da pesquisa. Houve distribuição e posterior recolhimento de questionário autoexplicativo. Os questionários foram previamente testados para que os dados obtidos fossem satisfatórios aos objetivos do trabalho, e também para que os participantes pudessem respondê-lo sem a orientação de um entrevistador. Consta de 20 perguntas, a maior parte de múltipla escolha, distribuídas em 16 itens (anexo); nenhum dos avaliados deixou de responder.

As informações contidas nos formulários foram mantidas em sigilo, preservando a identidade do entrevistado.

\section{RESULTADOS}

Os resultados são apresentados em forma de tabela.

A população-alvo é constituída por 202 mulheres e 117 homens entre 19 e 23 anos de idade, sendo que $62,7 \%$ fazem uso de correção óptica, principalmente para correção de miopia e astigmatismo miópico (51,7\%, Tabela 2). A maioria dos entrevistados teve a última consulta oftalmológica a menos de um ano (Tabela 1).

A tabela 3 demonstra o conhecimento sobre a cirurgia refrativa por parte dos estudantes. Dos entrevistados, $90 \%$ responderam já terem algum conhecimento sobre a cirurgia refrativa, sendo a fonte de informação, em ordem decrescente: familiares, amigos, faculdade e oftalmologista. Somente 109 $(34,2 \%)$ conheciam o procedimento cirúrgico; $229(71,8 \%)$ desconheciam os riscos e complicações do procedimento; e 260 $(81,5 \%)$ gostariam de receber mais informações sobre a cirurgia.

Dos entrevistados, cinco (1,5\%) já foram submetidos a essa operação e apresentam índice de satisfação muito grande, ao redor de $100 \%$. Contudo, os riscos e complicações pós-operatórias eram conhecidos por apenas dois $(40,0 \%)$ dos operados.

Apresentam interesse na realização da cirurgia refrativa $101(50,5 \%)$ dos usuários de correção óptica. As razões citadas da não inclusão na cirurgia foram, em ordem decrescente: contra-indicação médica e falta de oportunidade. Já os desinteressados relataram, como motivo mais comum, o baixo grau do vício de refração.

A cura definitiva pela cirurgia refrativa é a principal expectativa entre os usuários de correção óptica $(69,0 \%)$ como demonstrado na tabela 4 .

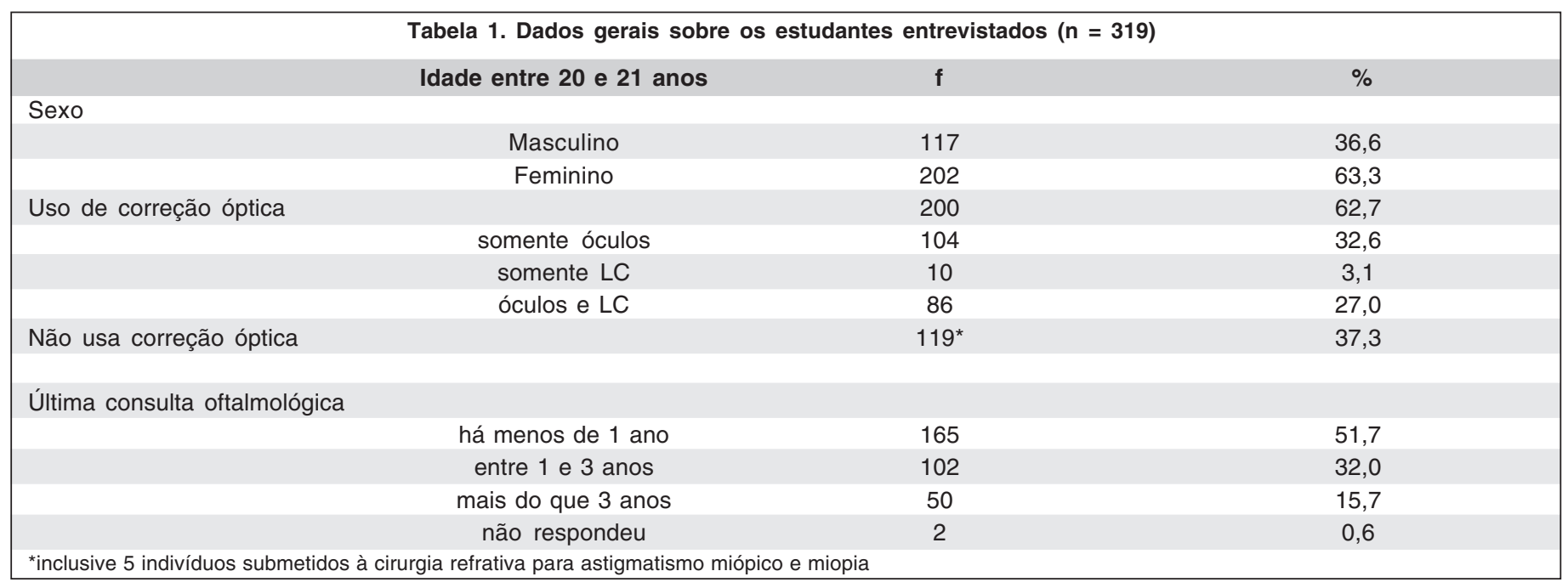




\begin{tabular}{|lcc|}
\hline \multicolumn{3}{|c|}{$\begin{array}{c}\text { Tabela 2. Problemas visuais apontados pelos estudantes } \\
\text { usuários de correção óptica (n=200) }\end{array}$} \\
& $\mathbf{f}$ & $\%^{*}$ \\
Miopia & 70 & 21,9 \\
Miopia e astigmatismo & 95 & 29,7 \\
Astigmatismo & 17 & 5,3 \\
Astigmatismo e hipermetropia & 11 & 3,4 \\
Hipermetropia & 5 & 1,5 \\
Não responderam & 2 & 0,6 \\
* O total é maior que 100\% por ser permitida mais de uma opção & \\
\hline
\end{tabular}

\section{DISCUSSÃO}

Dos 319 alunos entrevistados, $62,7 \%$ utilizavam algum tipo de correção óptica ${ }^{(5)}$. Estes achados vão de encontro com os dados encontrados na literatura a respeito da prevalência do uso de óculos entre estudantes de medicina ${ }^{(7)}$ que se mostraram pouco conhecedores sobre cirurgia refrativa; $92,8 \%$ relataram saber algo sobre a cirurgia, porém apenas $34,2 \%$ sabiam sobre procedimento cirúrgico e $17,6 \%$ tinham conhecimento dos riscos e complicações da cirurgia. Por outro lado, $81,5 \%$ gostariam de ser melhores informados em relação à correção cirúrgica dos vícios de refração.

Esta falta de informação sobre cirurgia refrativa numa população de estudantes de medicina, dos quais $62,7 \%$ são di- retamente interessados em correção óptica, reforça achados anteriores que mostram o desconhecimento em saúde ocular da população brasileira. Em uma análise entre 1000 entrevistados na cidade de Campinas ${ }^{(8)}$ e entre pacientes com glaucoma vistos pela primeira vez na Universidade de Campinas $^{(9)}$, foi enfatizado o desconhecimento em relação às doenças oculares mais prevalentes, até mesmo entre os portadores de glaucoma e ressaltaram a premência de projetos de educação em saúde pública como medida para evitar cegueira em nosso meio.

Ressalta-se, ainda, que $3(60,0 \%)$ dos 5 operados, também, tinham informações insuficientes a respeito dos riscos da cirurgia refrativa.

Dentre as fontes de informação sobre a cirurgia, o oftalmologista foi o quarto mais citado, precedido por familiares, amigos e faculdade, isto apesar de 267 (83,7\%) terem feito exame oftalmológico a menos de 3 anos. Este fato indica que a cirurgia refrativa estava sendo pouco divulgada e/ou analisada nos consultórios oftalmológicos, pois $260(81,5 \%)$ manifestaram interesse em maiores informações sobre o procedimento e muitos alegaram não ter se submetido à cirurgia por falta de informação, falta de confiança e/ou medo (Tabela 3).

Dos 200 amétropes, 138 (69,0\%) esperariam ficar totalmente livres do uso de óculos com a realização da cirurgia. Esta falsa expectativa, também, revela falta de conhecimento sobre os resultados da cirurgia, pois, apesar dos excelentes resulta-

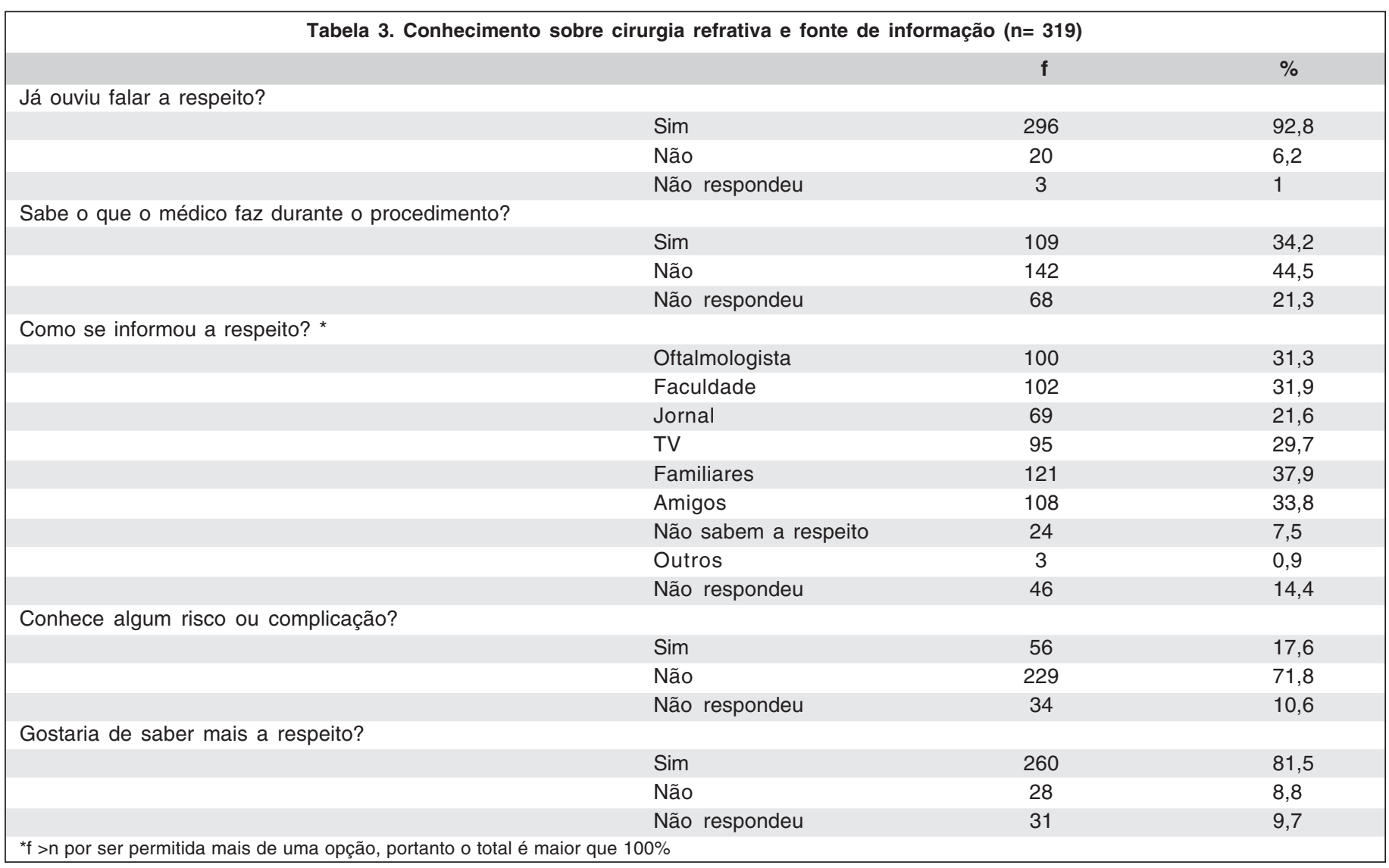




\begin{tabular}{|c|c|c|}
\hline \multicolumn{3}{|c|}{ Tabela 4. Expectativas sobre a cirurgia $(n=319)$} \\
\hline & f & $\%$ \\
\hline Cura definitiva & 138 & 69 \\
\hline Diminuição da dependência de correção & 21 & 10,5 \\
\hline Não respondeu & 40 & 20,5 \\
\hline \multicolumn{3}{|l|}{ Gostaria de ser operado? $(n=200)$} \\
\hline Sim & 101 & 50,5 \\
\hline Não & 74 & 74 \\
\hline Não responderam & 25 & 12,5 \\
\hline \multicolumn{3}{|l|}{ Se gostaria, por que ainda não foi? ${ }^{* *}$} \\
\hline Contra indicação médica & 40 & 39,6 \\
\hline Problemas financeiros & 25 & 24,7 \\
\hline Falta de confiança & 12 & 11,8 \\
\hline Falta de conhecimento & 28 & 27,7 \\
\hline Falta de oportunidade & 38 & 37,6 \\
\hline Medo dos resultados & 19 & 18,8 \\
\hline Outros & 10 & 9,9 \\
\hline Não responderam & 2 & 1,9 \\
\hline \multicolumn{3}{|l|}{ Se não gostaria, porquê?** } \\
\hline Baixo grau & 53 & 71,6 \\
\hline Falta de interesse & 16 & 21,6 \\
\hline Falta de confiança & 14 & 18,9 \\
\hline Falta de conhecimento & 12 & 16,2 \\
\hline Medo dos resultados & 13 & 17,5 \\
\hline Problemas financeiros & 5 & 6,7 \\
\hline Contra indicação médica & 2 & 2,7 \\
\hline Outros & 1 & 1,3 \\
\hline Não responderam & 5 & 6,7 \\
\hline
\end{tabular}

dos, essa cirurgia se propõe a diminuir ao máximo a necessidade de correção óptica, devendo os candidatos ser instruídos sobre os riscos cirúrgicos e sobre a impossibilidade de se garantir correção total ${ }^{(10)}$.

\section{CONCLUSÃO}

A maioria dos estudantes de Medicina $(62,7 \%)$ é portador de vício de refração corrigido, sendo os mais freqüentes a miopia simples e a miopia associada a astigmatismo. Há pouco conhecimento e falsa expectativa em relação à cirurgia, sendo que apenas $34,2 \%$ dos entrevistados conhecem o procedimento cirúrgico, $17,6 \%$ sabem dos riscos e complicações, e 69\% esperam cura total. Diante das condições desse estudo, foi constatado que apesar de muitos se interessarem pela cirurgia refrativa, poucos se submeteram a ela, devido, principalmente, em ordem decrescente, a: contraindicação médica, falta de oportunidade, falta de conhecimento e problemas financeiros. Os consultórios oftalmológicos são apenas a quarta fonte de informação, precedido, em ordem decrescente, por familiares, amigos e faculdade, o que é evidenciado pelo fato de $81,5 \%$ dos entrevistados gostariam de se informar melhor sobre a cirurgia refrativa. Mesmo tendo $83,7 \%$ dos entrevistados se consultado nos últimos três anos, informações e esclarecimento adequado não foram fornecidos.

\section{SUGESTÃO}

Baseado nos achados desta pesquisa sugere-se que os oftalmologistas forneçam mais esclarecimentos aos pacientes, de forma oral e/ou escrita sobre a cirurgia refrativa.

\section{AGRADECIMENTOS}

Dra. Edméa Rita Temporini, Yara Juliano, Lídia Cotait Kara-José.

\section{ABSTRACT}

Three hundred and nineteen students from the Medical School of ABC (Santo André-SP) responded to a self-evaluation questionnaire. $62.7 \%$ had optical correction, of these $53.3 \%$ showed simple myopia or myopia associated to astigmatism as refraction errors; $92.8 \%$ had heard about refractive surgery; $34.2 \%$ were aware of this surgical technique and only $17.6 \%$ knew about the risks including intra and post operative complications. Of the 200 students who showed to have ametropia, 50.5\% would like to be submitted to surgery and $69.0 \%$ expected to have recovery after surgery. Of all students, $51.7 \%$ had their last ophthalmological exam in less than a year; $32 \%$ were seen between 1 and 3 years and $15.7 \%$ in three years. Therefore, within the scope of this study, there has been little information about refractive surgery in ophthalmology clinics, staying far behind families, friends and university.

Keywords: Refraction, ocular; Refractive errors/surgery; Knowledge, attitudes, practice; Students, medical

\section{REFERÊNCIAS}

1. Abbott R, Asbell P, Driebe W, Elander R, Farber A. Low to moderate refractive errors. San Francisco: The American Academy of Ophthalmology; 1991. p.2.

2. Key II, James E. The Clao pocket guide to contact lens fitting. New York, Kellner /McCaffery; 1994. p.7.

3. Minguini N, Coelho RP, Serpa JF, Kara-José N, Holzchuh N. Características do uso dos óculos e lente de contato em uma comunidade universitária. Arq Bras Oftalmol 1994;57:126-8.

4. Sato T, Akiyama, Shibata H. A new surgical approach to myopia. Am J Ophthalmol 1953;36:826-9.

5 . Talamo JH, Krueger RR. The excimer laser manual: a clinician's guide to excimer laser surgery. New York: Little, Brown; 1997. p.19.

6. Waring GO. Quality of vision and freedom from optical correction after refractive surgery. J Refract Surg 1997;13:213-5.

7. Hansen C, Kristiansen T, Christoffersen T. High prevalence of myopia among medical students? Acta Ophthalmol Scand 1993;71:429.

8. Kara-José N, Saba HC, Cartoeci AA, Braga AC, Shimoda GA, Guide HC et al. Conhecimentos e práticas em saúde ocular de mil pessoas da cidade de Campinas Arq Bras Oftalmol 1995;48:160-4

9. Costa VP, Vasconcelos JPC, Pelegrino M, Kara-José N. O que os pacientes sabem sobre glaucoma? Arq Bras Oftalmol 1995;58:36-41.

10. Boyd B F. Atlas mundial de cirurgia oftalmológica-cirurgia refrativa da córneacirurgia refrativa com excimer laser. Highlights Ophthalmol 1997;4:4-5. 